\title{
Integrated care prevents hospitalisations for exacerbations in COPD patients
}

\author{
A. Casas*, T. Troosters ${ }^{+}$, J. Garcia-Aymerich*, J. Roca*, C. Hernández*, A. Alonso*, \\ F. del Pozo", P. de Toledo", J.M. Antó\#, R. Rodríguez-Roisín*, \\ M. Decramer ${ }^{+}$and members of the CHRONIC Project
}

ABSTRACT: Hospital admissions due to chronic obstructive pulmonary disease (COPD) exacerbations have a major impact on the disease evolution and costs. The current authors postulated that a simple and well-standardised, low-intensity integrated care intervention can be effective to prevent such hospitalisations.

Therefore, 155 exacerbated COPD patients (17\% females) were recruited after hospital discharge from centres in Barcelona (Spain) and Leuven (Belgium). They were randomly assigned to either integrated care (IC; $n=65$; age mean \pm SD $70 \pm 9 \mathrm{yrs}$; forced expiratory volume in one second (FEV 1$) 1.1 \pm 0.5 \mathrm{~L}, 43 \%$ predicted) or usual care (UC; $n=90$; age $72 \pm 9 \mathrm{yrs}$; FEV 1 $1.1 \pm 0.05 \mathrm{~L}, 41 \%$ pred). The IC intervention consisted of an individually tailored care plan upon discharge shared with the primary care team, as well as accessibility to a specialised nurse case manager through a web-based call centre.

After 12 months' follow-up, IC showed a lower hospitalisation rate $(1.5 \pm 2.6$ versus $2.1 \pm 3.1)$ and a higher percentage of patients without re-admissions (49 versus $31 \%$ ) than UC without differences in mortality (19 versus $16 \%$, respectively).

In conclusion, this trial demonstrates that a standardised integrated care intervention, based on shared care arrangements among different levels of the system with support of information technologies, effectively prevents hospitalisations for exacerbations in chronic obstructive pulmonary disease patients.

KEYWORDS: Chronic obstructive pulmonary disease, healthcare delivery, information technology, integrated care, planned care

hronic obstructive pulmonary disease (COPD) generates a significant burden on healthcare systems worldwide [1,2]. Hospital admissions due to exacerbations [3] are a major problem in the management of the disease due to their negative impact on health-related quality of life $[4,5]$, prognosis and costs $[6,7]$.

Unlike other chronic conditions [8,9], efficacy of interventions aimed at preventing hospitalisations due to exacerbations in clinically stable COPD patients is controversial [10-13]. While two randomised controlled trials $[12,13]$ failed to show positive effects, the Quebec study [10] reported effectiveness of patient empowerment for self-management in moderate-to-severe COPD. Similarly, positive results have been shown by GALLEFOSS [11] in mild-to-moderate COPD patients after 1-yr follow-up. Two main factors might explain the inconclusive findings on this issue, namely the heterogeneity of the

For editorial comments see page 4 patients examined and the insufficient standardisation of the interventions in these studies.

It can be reasonably assumed that together with the severity of the disease [2], concomitant factors, such as comorbidities, patient's anxiety/ depression [14], uncovered social needs and poor self-management of the disease, may modulate hospitalisation rates in these patients. In this scenario, it was hypothesised that a simple, welldefined integrated care (IC) intervention [15, 16], with the support of information and communication technologies (ICT), may be effective to prevent hospitalisations for exacerbations in COPD patients. The novelty of the current study's approach is that the preventive intervention relied on shared-care arrangements between primary care teams and hospital teams, which aimed to avoid duplicates and to generate synergies among different levels of the healthcare system.

The current study was conducted in two cities, Barcelona (Spain) and Leuven (Belgium), with
AFFILIATIONS

*Servei de Pneumologia and Technology Innovation Unit, Hospital Clínic, IDIBAPS, Universitat de Barcelona

${ }^{\#}$ Respiratory and Environmental Health Research Unit, Institut Municipal d'Investigació Mèdica (IMIM-IMAS), Universitat Pompeu Fabra, Barcelona, and

- Grupo de Bioingeniería y

Telemedicina (GBT-UPM)

Universidad Politécnica de Madrid, Madrid, Spain.

${ }^{+}$Respiratory Division, UZ-Leuven,

Faculty of Kinesiology and

Rehabilitation, KU-Leuven, Belgium.

CORRESPONDENCE

J. Roca

Servei de Pneumologia

Hospital Clínic

Villarroel 170

Barcelona-08036

Spain

Fax: 34932275455

E-mail: jroca@clinic.ub.es

Received

May 312005

Accepted after revision:

March 252006

SUPPORT STATEMENT

The present study was supported by: the CHRONIC project (IST-1999/

12158) from the European Union; Marato de TV3; Comissionat per a Universitats i Recerca de la

Generalitat de Catalunya (SGR00386); Red Respira Instituto de Salud Carlos III (ISCIII)-Redes Temáticas de Investigación Cooperativa (RTIC)-03/11; and Red Telemedicina ISCIII-RTIC-03/117. A Casas and T. Troosters were research fellows supported by CHRONIC (IST1999/12158). T. Troosters is currently a postdoctoral research fellow of the Fonds voor Wetenschappelijk Onderzoek (Vlaanderen, Belgium). J. GarciaAymerich was supported by Red Respira (RTIC C03/11) and ICS III.

European Respiratory Journal Print ISSN 0903-1936 Online ISSN 1399-3003 
marked differences in the primary care settings. Consequently, the intervention required customisation to country specificities, particularly regarding the interactions between hospital and primary care teams. While the general practitioners (GPs) played a key role in Leuven, the intervention in Barcelona was essentially carried out by specialised nurses.

\section{METHODS}

\section{Selection and description of participants}

The current prospective controlled study was carried out in 155 COPD patients recruited into two tertiary hospitals (Hospital Clínic, Barcelona and University Hospital Gasthuisberg, UZLeuven, Leuven) immediately after the patients' hospital discharge. All of the patients were admitted because of a previous episode of exacerbation requiring hospitalisation for $>48 \mathrm{~h}$. Up to 850 COPD patients were screened, but only $19 \%$ fulfilled the defined criteria and were finally included for randomisation. Exclusion criteria for the study were: 1) not living in the healthcare area $(39 \%) ; 2$ ) severe comorbid conditions, i.e. lung cancer or other advanced malignancies, and extremely severe neurological or cardiovascular disorders $(25 \%)$; 3) logistical limitations due to extremely poor social conditions, such as illiteracy or no phone access at home (10\%); and 4) being admitted to a nursing home $(7 \%)$. All participants were informed in detail of the characteristics of the study and written informed consent was obtained in accordance with the Committee on Investigations Involving Human Subjects at the two centres, which approved the study. Only five $(3 \%)$ patients out of 160 did not accept to participate in the study.
All 155 patients (Barcelona $n=113$, Leuven $n=42$ ) included in the study were blindly assigned (1:1 ratio) using computergenerated random numbers to either IC or usual care (UC). As a different randomisation ratio was used in one of the centres (Barcelona 1:2 ratio) during part of the study, the interventions (IC and UC) had different numbers of patients $(n=65$ and $n=90$, respectively). The follow-up period was prolonged for $1 \mathrm{yr}$.

Figure 1 displays a patient's flow throughout the study. The primary end-point was hospital re-admission. In the two centres, re-hospitalisation was assessed through hospital records and systematic questionnaires on use of healthcare resources administered to patients as described in detail below. Mortality and utilisation of healthcare resources were also assessed.

\section{IC intervention}

The IC intervention was standardised for both centres, although slightly adapted to the two different healthcare systems and local geographical conditions. It included four key features. First, a comprehensive assessment of the patient at discharge, including severity of the respiratory disease, evaluation of comorbid conditions and analysis of requirements in terms of social support. Secondly, an educational programme on self-management of the disease administered at discharge, as described below. Thirdly, agreement on an individually tailored care plan following international guidelines $[2,17]$ was shared across the system via interaction between the specialised nurse case manager and the primary care team. Fourthly, accessibility of the specialised nurse to

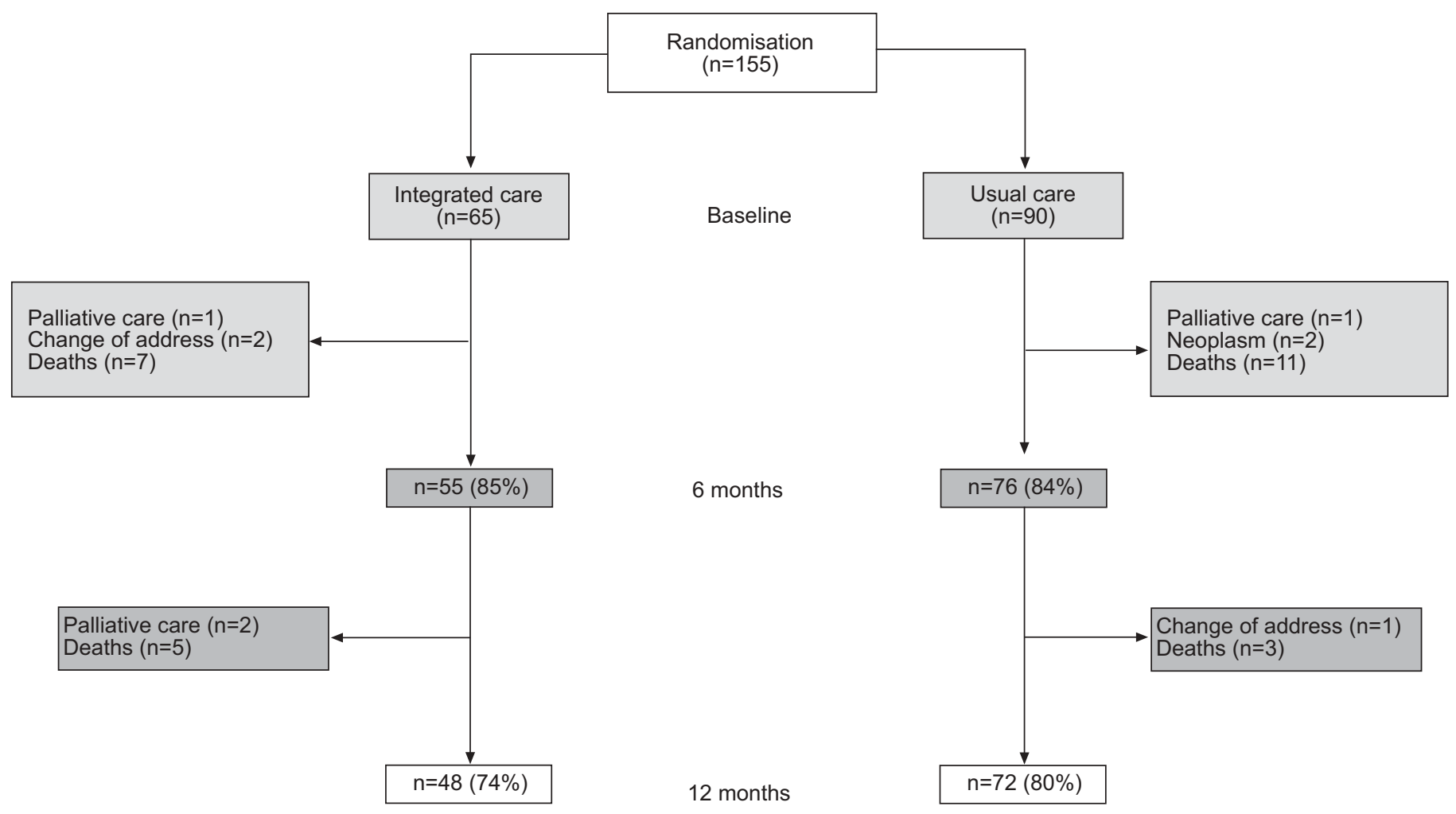

FIGURE 1. Schematic representation of the study profile over $1 \mathrm{yr}$. In total, 155 chronic obstructive pulmonary disease patients were included in the study. The patients were randomly assigned to integrated care or to usual care. The flow chart shows that all drop-outs during the follow-up were due to either exclusion criteria or death. 
patients/carers and primary care professionals during the follow-up period was ensured through an ICT platform including a web-based call centre (CHRONIC project (an Information Capture and Processing Environment for Chronic Patients in the Information Society), IST- 1999/12158).

In both hospitals, all patients in the IC arm of the study (together with their carers, if needed), undertook a comprehensive educational programme of $\sim 2 \mathrm{~h}$ duration immediately before discharge, which was administered by a specialised respiratory nurse. This programme covered several items, including knowledge of the disease, instructions on nonpharmacological treatment, assessment of administration techniques for pharmacological therapy and empowerment for selfmanagement of the disease, including strategies to adopt during future exacerbations [15, 17]. In each IC patient, the therapeutic plan was customised to the individual fragility factors [15] described below, and was shared with the primary care team. Reinforcement of the logistics for treatment of comorbidities and social support was carried out accordingly.

A different logistic approach was undertaken in the two study sites due to both geographical factors and specificities of the primary care structure. In Barcelona, one joint visit of the specialised nurse and the primary care team (physician, nurse and social worker) was performed at the patient's home within $72 \mathrm{~h}$ of discharge. During the follow-up period, the specialised nurse played the role of case manager.

In Leuven, the GPs planned regular visits to chronic patients, usually at home. The current study aimed at improving these visits regarding COPD care. It is important to realise that each patient in Leuven was allocated to a different primary care physician. GPs were invited to participate in regional education sessions aimed at enhancing knowledge on transmural care for patients with COPD (including information on the current study). Written information brochures on standard treatment plans and treatment guidelines for patients with COPD were sent to the primary care physicians. More importantly, the senior investigator in Leuven (M. Decramer) contacted the individual general practitioner (GP) of patients included in the treatment group. During this contact the treatment plan and points of attention in the healthcare management plan were discussed based on the individual patient files and identified points of fragility.

At both sites, weekly phone calls during the first month after discharge were carried out to reinforce self-management strategies. Phone calls were carried out after 3 and 9 months to inquire about utilisation of healthcare resources with no educational intervention. It is important to note that the IC intervention did not include further scheduled visits by the programme team during the follow-up period in either site. Nonscheduled visits could be triggered by the patients or their carer through the call centre.

\section{The chronic platform}

The chronic platform [18] facilitated accessibility of patients / carers and primary care professionals to the specialised nurse case manager. The central component of this ICT platform was a call centre coupled to a web-based application that provided access to and facilitated the management of records of the patients included in the programme. Technical and functional details of the ICT platform are reported elsewhere [18].

\section{UC intervention}

Patients included in the UC arm were discharged from hospital by the attending physician who decided on the outpatient control regime. Pharmacological prescriptions at discharge and in-hospital treatment followed the standard protocols of the centres involved in the study, which were similar in IC and UC $[2,17]$. Patients in the UC arm of the study were visited by their own physician without additional support. Visits were usually scheduled every 6 months. Specifically, the controls did not receive help from the specialised nurse, nor were they included in the educational programme or had access to the call-centre.

\section{Measurements and instruments}

Early assessment of patients at study admission was identical for both groups. Assessment included a blind administration of a questionnaire, described in detail elsewhere [15, 19], concerning: 1) risk factors for exacerbation (vaccination, smoking habits, comorbidities); 2) health-related quality of life status during the previous year (Saint George Respiratory Questionnaire and Euroqol); 3) history of previous exacerbations requiring in-patient hospitalisations and/or emergency room admissions (information was validated by examination of individual clinical records); 4) clinical features of the current exacerbation; 5) fragility factors, such as comorbid conditions, anxiety/depression, educational level, economic status and caregiver support [15]; and 6) treatment, including compliance, observed skills for administration of inhaled drugs, oxygen and physical activity. Vital signs, chest radiography films and pulmonary function tests (including arterial blood gases) were obtained in all patients upon admission.

Assessment of the use of healthcare resources by phone or personal interview was carried out at 1, 3, 6, 9 and 12 months in both arms of the study. Data regarding admissions during follow-up were obtained from hospital records. Data regarding mortality were obtained from hospital records and direct family interviews.

\section{Statistics}

Sample size calculation was obtained considering an IC:UC ratio of $1: 1.5$; thus, estimating a proportion of $60 \%$ admitted in the UC group and accepting an alpha risk of 0.05 and a beta risk of 0.20 in a two-sided test. A sample size of 60 IC patients and 90 UC patients was necessary to recognise a statistically significant relative risk of readmission $\geqslant 1.4$ in the UC versus the IC group. A drop-out rate of 0.15 was anticipated.

Results are expressed as mean $\pm \mathrm{SD}$, median (25th percentile75th percentile), or as percentages in the corresponding categories. Comparisons between UC and IC on admission were performed using independent unpaired t-tests, a nonparametric Mann-Whitney U-test or the Chi-squared test. All analyses were conducted according to the intention-to-treat principle. Sequential survival analysis was performed with a Cox proportional-hazards model to determine whether IC assignment was an independent predictor of hospitalisation after adjustment for variables that were different at baseline, i.e. hospital respiratory admissions in the previous year and 
influenza vaccination. Data regarding patients who died without re-admission were censored at the time of death. All analyses were carried out separately in each centre and also with pooled data. The use of healthcare resources was expressed as rate of re-admissions calculated as the number of re-admissions during the follow-up times (number of follow-up days/365). The difference in rate of re-admissions per year is expressed as the number of re-admissions during the follow-up year minus the number of re-admissions in the previous year. Survival without re-admission corresponds to subjects with no re-admissions among survivors. Differences between IC and UC were examined using the Kruskal-Wallis tests. Statistical significance was accepted at $\mathrm{p}<0.05$.

\section{RESULTS}

A total of 155 COPD patients who were discharged from two tertiary hospitals (Barcelona and Leuven; fig.1) were included in the analysis ( $\mathrm{IC}=65$ and $\mathrm{UC}=90$ ). Drop-outs for the entire follow-up period are described in figure 1 . As indicated in table 1, at entry, the patients of the two interventions showed similar characteristics except for the number of hospital admissions in the previous year $(1.0 \pm 1.3$ versus $0.64 \pm 1.2$ in the IC and UC group, respectively; $\mathrm{p}=0.05)$ and influenza vaccination ( 56 versus $78 \%$, respectively; $p=0.01$ ).

Since only patients with COPD and lack of very severe comorbidities or extreme social problems were considered eligible for the current study, the current authors compared relevant variables between participants and subjects excluded from the current study. The analysis showed that subjects excluded from the study had more chance of being female $(p=0.011)$ and older (mean difference 2 yrs; $p=0.02)$, and less chance of being smokers $(p=0.002)$ than the participants. No differences in the remaining variables, including intensive care unit admissions, were observed.

\section{Main outcomes}

IC was associated with a lower re-hospitalisation rate during follow-up than UC. The hazard ratio (HR) estimated by adjusted Cox analysis was 0.55 (95\% confidence interval (CI) $0.35-0.88 ; \mathrm{p}=0.01$ ) for Barcelona and Leuven as analysed jointly (fig. 2). The figure also displays a separate analysis of the two sites, showing similar results between Barcelona (HR 0.52 (95\% CI 0.28-0.95); $\mathrm{p}=0.04)$ and Leuven (HR 0.35 (95\% CI $0.15-0.80) ; \mathrm{p}=0.01)$.

The main outcome variables of the analysis with pooled data are displayed in table 2 , indicating that IC clearly presented a lower number of re-hospitalisations than UC $(p=0.02)$. The remaining variables in the table further support the contention that IC prevented hospitalisations compared with UC. The rate of readmissions ( 1.5 versus $2.1, p=0.033$ ) were significantly lower in the IC intervention than in UC. Likewise, the percentage of patients without admissions among survivors was also greater in IC $(49 \%)$ than in UC $(31 \%$; $=0.03)$. No significant differences regarding deaths were observed in IC and UC, 19 and 16\%, respectively.

The difference in rate of admissions per patient between the follow-up and the previous year was also lower in IC $(0.47 \pm 2.4)$ than in UC $(1.84 \pm 3.95 ; p=0.03)$.

\begin{tabular}{|c|c|c|c|}
\hline & IC & UC & p-values \\
\hline Subjects & 65 & 90 & \\
\hline \multicolumn{4}{|l|}{ Sociodemographics } \\
\hline Age yrs & $70 \pm 9$ & $72 \pm 9$ & 0.09 \\
\hline Sex (females) & $15(23)$ & $11(12)$ & 0.64 \\
\hline Education less than primary & $16(25)$ & $20(22)$ & 0.85 \\
\hline Annual income $<€ 6000$ & $7(13)$ & $16(24)$ & 0.16 \\
\hline \multicolumn{4}{|l|}{ Clinical and functional profile } \\
\hline Length baseline hospitalisation & $8.5 \pm 5.5$ & $7.8 \pm 4.2$ & 0.65 \\
\hline MRC dyspnoea scale & $3.5 \pm 1.3$ & $3.5 \pm 1.2$ & 0.84 \\
\hline BMl $\mathrm{kg} \cdot \mathrm{m}^{-2}$ & $26.4 \pm 5.1$ & $26.1 \pm 5.4$ & 0.71 \\
\hline FVC \% pred & $64 \pm 21$ & $63 \pm 18$ & 0.79 \\
\hline FEV $1 \%$ pred & $43 \pm 20$ & $41 \pm 15$ & 0.89 \\
\hline $\mathrm{FEV}_{1} / \mathrm{FVC} \%$ & $48 \pm 18$ & $48 \pm 17$ & 0.95 \\
\hline $\mathrm{Pa}, \mathrm{O}_{2} \mathrm{mmHg}$ & $64 \pm 11$ & $64 \pm 14$ & 0.65 \\
\hline $\mathrm{Pa}, \mathrm{CO}_{2} \mathrm{mmHg}$ & $44 \pm 7$ & $44 \pm 7$ & 0.87 \\
\hline Comorbidities & $1.9 \pm 1.4$ & $1.8 \pm 1.5$ & 0.74 \\
\hline $\begin{array}{l}\text { Hospital respiratory admissions } \\
\text { previous yr }\end{array}$ & $1.0 \pm 1.3$ & $0.6 \pm 1.2$ & 0.05 \\
\hline SGRQ total score & $57 \pm 18$ & $59 \pm 18$ & 0.46 \\
\hline Euroqol analogue visual scale ${ }^{\#}$ & $47.8 \pm 18.1$ & $47.4 \pm 18.5$ & 0.87 \\
\hline Anxiety and depression Goldberg score & $8.5 \pm 5.6$ & $8.2 \pm 5.9$ & 0.71 \\
\hline Apgar score & $7.6 \pm 4.2$ & $6.3 \pm 2.9$ & 0.18 \\
\hline \multicolumn{4}{|l|}{ Regular COPD treatment } \\
\hline LTOT & $16(25)$ & $21(23)$ & 0.85 \\
\hline Influenza vaccination & $36(56)$ & $68(78)$ & 0.01 \\
\hline Pneumococcal vaccination & $19(36)$ & $41(53)$ & 0.07 \\
\hline Home rehabilitation & $17(26)$ & $20(22)$ & 0.57 \\
\hline \multicolumn{4}{|l|}{ Self-management } \\
\hline Active smokers & $21(32)$ & $19(21)$ & 0.14 \\
\hline $\begin{array}{l}\text { Knowledge-name and cause of the } \\
\text { disease }\end{array}$ & $45(69)$ & $58(65)$ & 0.61 \\
\hline Poor adherence to inhalers & $14(23)$ & $23(28)$ & 0.56 \\
\hline Regular physical activity & $56(86)$ & $82(91)$ & 0.43 \\
\hline Knowledge alarm symptoms and drugs & $44(68)$ & $66(73)$ & 0.48 \\
\hline
\end{tabular}

Data are presented as $n$, mean $\pm S D$ or $n(\%)$. IC: integrated care; UC: usual care; MRC: Medical Research Council scale for scoring dyspnoea; BMI; body mass index; FVC: forced vital capacity; \% pred: \% predicted; FEV1: forced expiratory volume in one second; $\mathrm{FEV}_{1} / \mathrm{FVC}$ ratio: expressed as actual value; $\mathrm{Pa}_{\mathrm{a}} \mathrm{O}_{2}$ : arterial oxygen tension; $\mathrm{Pa}_{\mathrm{a}} \mathrm{CO}_{2}$ : carbon dioxide arterial tension; $\mathrm{SGRQ}$ : Saint George's Respiratory Questionnaire to assess health-related quality of life; Apgar: adaptability, partnership, growth, affection and resolve; COPD: chronic obstructive pulmonary disease; LTOT: long-term oxygen therapy. " : instrument to assess health-related quality of life; $"$ : to assess family functionality. 1 $\mathrm{mmHg}=0.133 \mathrm{kPa}$.

\section{Use of healthcare resources}

Table 3 displays the main data on utilisation of both scheduled and nonscheduled services in the two sites. No significant differences were observed between IC and UC in the number of visits. It is of note that all results in table 3 are expressed as median (25th percentile-75th percentile). In Barcelona (IC $=44$ ), a total of 448 phone calls through the call centre, $194(43 \%)$ scheduled and 254 unscheduled, were carried out during the 12-month follow-up period. In Leuven $(\mathrm{IC}=21)$, the total number of phone calls during the study was 201, $128(63 \%)$ 

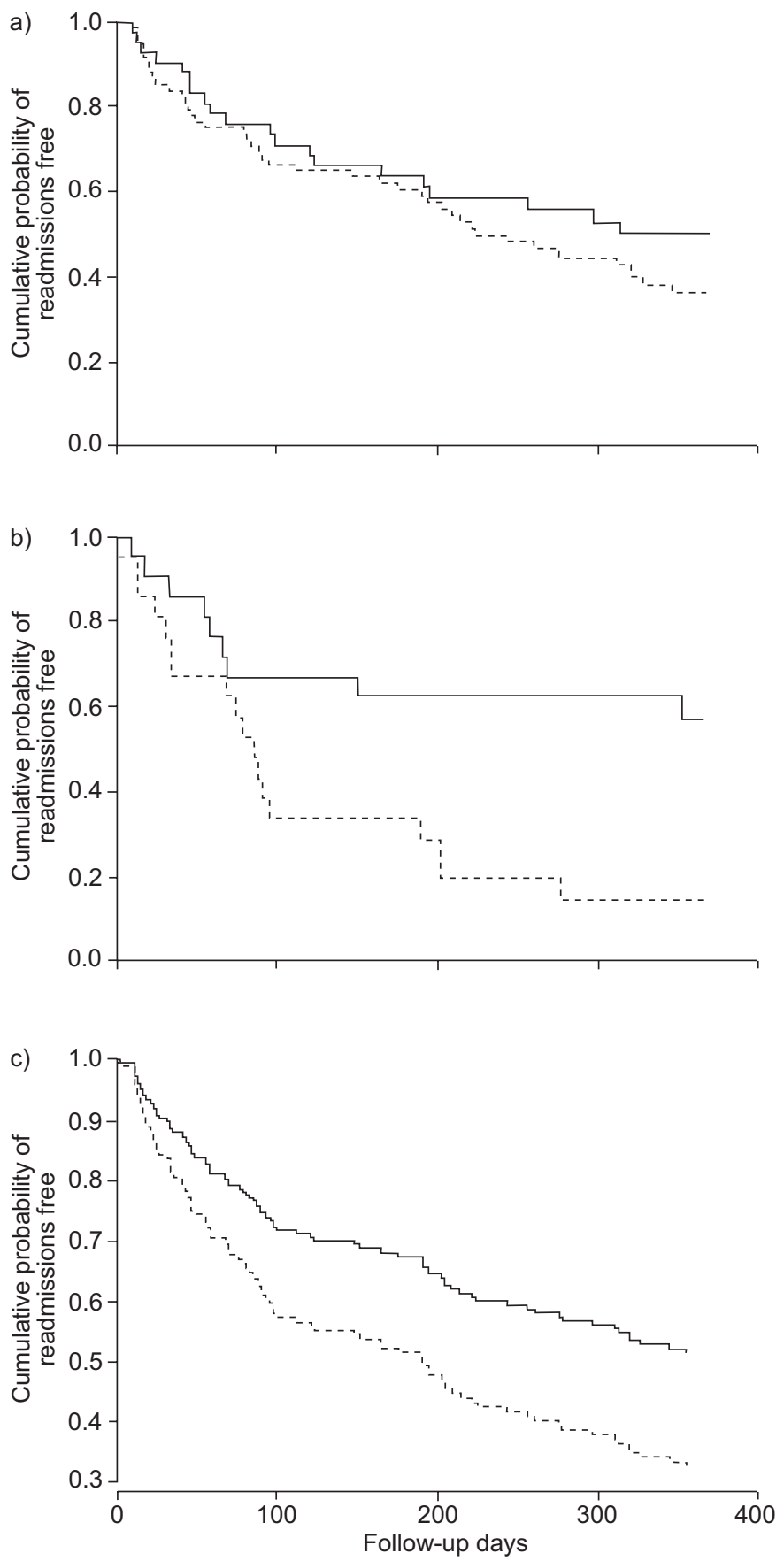

FIGURE 2. The results of the Cox analyses carried out separately in a) Barcelona and b) Leuven. c) The results of the analysis carried out with pooled data in both centres. In all cases, chronic obstructive pulmonary disease patients in integrated care (-) showed a higher rate of admission-free time than usual care (- - -). a) Hazard ratio (HR) 0.52 (95\% confidence interval (Cl) 0.28-0.97), p=0.04; b) HR 0.35 (95\% Cl 0.15-0.80), $p=0.01$; c) HR 0.55 (95\% Cl 0.34-0.87), $p=0.01$.

scheduled and 73 unscheduled. In this centre, only five (22\%) out of the 22 patients used the service by triggering the phone call. Fourteen $(67 \%)$ out of the 21 patients in the IC intervention received unscheduled phone calls by the specialised nurse. Unscheduled phone calls were mostly related to worsening of symptoms (patient-triggered) or follow-up of exacerbations

\begin{tabular}{|c|c|c|c|}
\hline & IC & UC & p-value \\
\hline Subjects & 65 & 90 & \\
\hline \multicolumn{4}{|l|}{ Number of readmissions } \\
\hline 0 & $36(55)$ & $30(33)$ & 0.028 \\
\hline 1 & $14(22)$ & $34(38)$ & \\
\hline$\geqslant 2$ & $15(23)$ & $26(29)$ & \\
\hline $\begin{array}{l}\text { Number of readmissions during } \\
\text { follow-up year }\end{array}$ & $0.9 \pm 1.3$ & $1.3 \pm 1.7$ & 0.028 \\
\hline $\begin{array}{l}\text { Rate of readmissions during } \\
\text { follow-up year }\end{array}$ & $1.5 \pm 2.6$ & $2.1 \pm 3.1$ & 0.033 \\
\hline $\begin{array}{l}\text { Difference on rate of re-admissions } \\
\text { per year }\end{array}$ & $0.5 \pm 2.6$ & $1.5 \pm 3.1$ & 0.003 \\
\hline Survival without readmissions & $32(49)$ & $28(31)$ & 0.03 \\
\hline Total deaths & $12(19)$ & $14(16)$ & 0.67 \\
\hline \multicolumn{4}{|c|}{$\begin{array}{l}\text { Data are presented as } n, n(\%) \text { or mean } \pm S D \text {. IC: integrated care; UC: usual } \\
\text { care. Rate of re-admissions during the follow-up year was calculated as the } \\
\text { number of re-admissions during the follow-up } \times \text { number of follow-up days } / 365 \text {. } \\
\text { The difference in rate of re-admissions per year is expressed as the number of } \\
\text { re-admissions during the follow-up year minus the number of re-admissions in } \\
\text { the previous year. Survival without re-admission corresponds to subjects with } \\
\text { no re-admission among survivors. }\end{array}$} \\
\hline
\end{tabular}

(nurse-triggered). The mean duration of the phone calls in the two sites was $\sim 20 \mathrm{~min}$. In Leuven, no home visits by hospitalbased specialised nurses were planned after discharge.

\section{DISCUSSION}

The present study demonstrates the effectiveness of a welldefined low-intensity IC programme in COPD patients to prevent future exacerbation episodes triggering hospital admissions. In the current study, three major novel aspects were identified: 1) standardisation of the IC intervention; 2) pilot development of a distributed model with shared arrangements between primary care, hospital and community services; and 3) use of ICT as a supporting tool for the programme. It must be highlighted that the effects of the IC intervention were similar in both countries. The intervention was customised to the organisational specificities of primary care in the two participating countries, but its most distinctive feature was common in both sites, that is, to promote standardised shared-care arrangements between levels of care to prevent COPD exacerbations.

In the current study, as opposed to previous reports on prevention of hospitalisations for exacerbations in COPD patients $[10,12]$, the educational programme was only one of the components of the IC strategy. The effectiveness of IC can be explained by different concomitant factors. It can be reasonably assumed that the intervention resulted in enhanced self-management of the disease together with a higher accessibility to healthcare professionals. Likewise, the potential impact of the intervention on enhanced management of comorbid conditions and social problems cannot be neglected. Altogether the interventional impact may have prompted an early detection and better management of exacerbations in the IC programme. A recent report by WILKINSON et al. [20] 
indicates that patient recognition of exacerbation symptoms and prompt treatment in COPD patients improves exacerbation recovery and reduces risk of hospitalisation. It is also associated with a better health-related quality of life.

Preliminary analyses of other follow-up variables in a subsample of these patients, not reported in the current study, showed no differences in smoking habits or physical activity practice at 12 months between UC and IC. However, it is of note that all variables related to COPD knowledge, selfmanagement and adherence to treatment were better scored in IC than in UC. No differences between groups were found in pharmacological therapies, physical rehabilitation or in patient satisfaction.

\section{Internal and external validity of the trial}

Adequacy of the assignment process to either IC or UC was ensured by both the generation of the allocation sequence by a random process and preventing foreknowledge of the treatment assignments in the specialised team that implemented the allocation sequence. As described previously, the different randomisation ratio in the two centres provoked an imbalance in the number of patients assigned to each arm of the study $(\mathrm{IC}=65$ and $\mathrm{UC}=90)$. The ratio 1:2 was transiently used in the Hospital Clínic (Barcelona) as a conservative approach to ensure appropriate logistics for the study. The allocation sequence alluded to above cannot explain the differences between IC and UC at entry of the study (table 1) observed in Barcelona. As indicated previously, the statistical analysis included adjustment for variables which were different at baseline (number of hospital admissions in the previous year and influenza vaccination). Moreover, the consistency of the results in Barcelona was further ensured by examining the history of hospitalisations in the previous 2 yrs for both IC and UC. A strength of the present analysis was that there were no subjects lost to follow-up, since all drop-outs were due to appearance of exclusion criteria or death (fig. 1) and, in any case, valid information about re-hospitalisations was available from the national health services.

Despite the IC intervention being adapted to the specificities of the healthcare system in each site, it showed, as a common and most distinctive feature, the development of planned sharedcare arrangements between levels of care. In Barcelona specifically, one joint visit of the specialised nurse and the primary care team at the patient's home was carried out within $72 \mathrm{~h}$ of discharge. This approach was chosen because of its practicality, as the hospital is the reference centre of a geographically small, but highly populated $(500,000$ inhabitants) urban sector. In contrast, the UZ-Leuven centre covers a large geographical area and patients live in remote areas away from the hospital, making the initial joint visit to the patient's home impractical. In addition, GPs in Belgium are stimulated to play a role as "gate keepers" of the healthcare system.

The initial joint visit in Barcelona was likely to be useful for implementation of individualised care plans shared by patients/care givers, primary care professionals and the hospital team. In Leuven, the beneficial effects of the intervention should also be attributed to the planned shared care arrangements between hospital-based professionals and primary care doctors, as described above. Since the Leuven site

\begin{tabular}{|c|c|c|c|c|}
\hline \multirow[t]{2}{*}{ TABLE 3} & \multicolumn{4}{|c|}{$\begin{array}{l}\text { Use of healthcare resources }{ }^{\#} \text { (rate per year) } \\
\text { during the follow-up }\end{array}$} \\
\hline & & IC & uC & p-value \\
\hline Subjects & & 65 & 90 & \\
\hline \multicolumn{5}{|c|}{ Follow-up time days } \\
\hline Barcelona & & $297 \pm 115$ & $319 \pm 100$ & 0.088 \\
\hline Leuven & & 365 & 365 & \\
\hline \multicolumn{5}{|c|}{ Nurse home visits within the programme } \\
\hline Barcelona & & $3(1-10)$ & & \\
\hline Leuven & & NP & & \\
\hline \multicolumn{5}{|l|}{ Doctor visits } \\
\hline Barcelona & & $2(0-4)$ & $2(1-4)$ & 0.437 \\
\hline Leuven GP & at home & $10(7-18)$ & $13(9-27)$ & 0.454 \\
\hline \multicolumn{5}{|c|}{ Phone calls patient-triggered } \\
\hline Barcelona & & $2(0-8)$ & & \\
\hline Leuven & & $0(0-0)$ & & \\
\hline \multicolumn{5}{|c|}{ Phone calls follow-up intervention } \\
\hline Barcelona & & $1(1-3)$ & & \\
\hline Leuven & & $2(0-3)$ & & \\
\hline \multicolumn{5}{|c|}{ 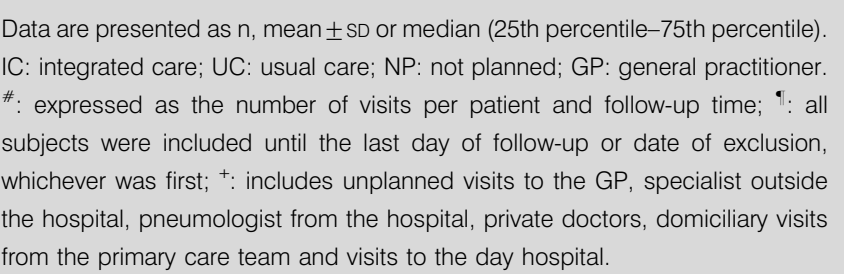 } \\
\hline
\end{tabular}

did not show statistical significant differences in the number of GP visits (table 3), it is reasonable to assume that the intervention enhanced the quality of these visits, leading to improved clinical outcomes as seen in the IC group (fig. 2). In other words, the beneficial effects of the IC intervention did not seem to be associated with the number of home visits carried out either by a nurse (Barcelona) or a primary care physician (Leuven).

In the current study, the outcomes of the IC intervention after stratification by disease severity were not reported. The latter should be addressed considering different covariates, namely Global Initiative for Chronic Obstructive Lung Disease classification [2], history of previous exacerbations and comorbid conditions not leading to exclusion criteria. This assessment was not done partly because of insufficient statistical power of the study for this specific purpose. However, it is of note that the current disease-orientated programme only allowed inclusion of 155 COPD patients. This figure represents 452 (34\%) of all screened COPD patients living in the healthcare sector and willing to participate in the study. It can be reasonably assumed that no selection bias was present due to exclusion of those patients not living in the healthcare sector $(n=331)$. A rough revision of the current underlying reasons for exclusion indicates that most of the 452 COPD patients would have been eligible for properly tailored patient-centred IC programmes. It can be argued that a patientfocused strategy instead of the current disease-orientated approach must be adopted to properly fulfil the principal aims of the Chronic Care Model [21, 22]. In the present study, 
an IC pathway for COPD has been successfully applied, but from the screening data it has become clear that patients with cancer, severe neurological or cardiovascular disorders $(n=212)$, or those who were indigent $(n=145)$ required a specific integrated pathway either across several medical specialities and/or appropriate social support, respectively. The approach adopted in the current study may limit its external validity, as in most clinical trials by definition, but increases internal validity. Given the very small number of nonresponses (five subjects, $3 \%$ of the eligible), the current authors' opinion is that eligibility criteria, although restrictive, allow for a highly valid estimation of the effect of an IC programme in COPD patients without severe comorbidities. In other words, the study design reinforces the present author's conclusion on the beneficial effects of IC to prevent hospitalisations in the COPD patients studied, but the results cannot be generalised to all elderly COPD patients with very severe comorbid conditions admitted to the emergency room because of an episode of exacerbation.

Analysis of the economical implications of the intervention was beyond the scope of the present study. However, it is of note that the outpatient consultations outside of the IC programme were similar between both arms of the study. Likewise, the number of home visits in the IC group and the requested activity through the call centre during the 12-month follow-up were rather modest. The total number of phone calls per patient through the call centre was similar in both countries. However, only a small percentage of patients in Leuven (24\%) triggered phone calls, which was not the case in Barcelona. This phenomenon had no implication on outcomes and it can be explained by differences in primary care between Belgium and Spain. Overall, the present authors understand that the extension of the IC intervention has a clear potential for cost-containment because of the significant savings generated in both direct and indirect costs due to prevention of hospitalisations. Moreover, the homogeneity of the outcomes in the two sites, together with the simplicity of the intervention, indicates that the proposed strategy shows potential for a successful deployment in other healthcare systems.

\section{IC and information technologies}

The conceptual aspects of planned care for chronic patients were originally formulated by WAGNER [21] and were subsequently adopted by the World Health Organization [22]. Encouragement of preventive strategies promoting behavioural changes in life style and the design of innovative home-based services must become components of integrated care strategies within regional networks, by no means confronted to patient hospitalisation. In building up such strategies, the establishment of effective functional relationships among levels of care will preclude the current fragmentation of services. Activities included in the integrated interventions explored in the current study cannot easily be made operative in the absence of a robust ICT support.

In conclusion, the integrated care intervention proposed in the current study significantly improved the management of moderate-to-severe chronic obstructive pulmonary disease patients and facilitated the logistics for an appropriate longterm follow-up of these patients. The current authors understand that the prevention strategy examined in the present study constitutes a valid contribution to be considered in future versions of the chronic obstructive pulmonary disease guidelines $[2,17]$.

\section{ACKNOWLEDGEMENTS}

The authors would like to thank N. Soler, J. Fernandez, R. Estruch and D. Estrada. They would also like to thank F. Mota from the Hospital Clínic (Barcelona, Spain) for his support during the study. The authors also acknowledge the support of X. Esteva, D. Martí, M. Esquerdo, L. Lozano, and M. Amor (Programes d'Atenció Domicilària-Equips de Suport), A. Garrigos (Fundació Conviure), C. Olmos (Centre d'Atenció Primària, Manso), and M. Borrell (Centre d'Atenció Primària, Sarria).

The authors are grateful to all the partners of the CHRONIC Project. J.A. Montero, B. Vallespin, J. Pastor and B. Sifre (Corporacìo Sanitaria Clinic, Barcelona, Spain); S. Jiménez (Univerdidad Politecnica, Madrid, Spain); F. Ortiz, L. Mena, M. Godoy, J.L. Sirera (Centro Español de Servicios Telemáticos, Madrid, Spain); J. Aguiló, L. Sánchez, A. Guimerà (Consejo Superior de Investigaciones Científicas-Centro Nacional de Microelectrónica, Barcelona, Spain); B. Andrés, J.J. Moratillas (SMS, Madrid, Spain); M.A. García Matatoros, A. Marcus (Alamo-on-line, Madrid, Spain); R. Farré (UB, Barcelona, Spain); C. Céinos (Ecomit Consulting, Barcelona, Spain); A. Falco, D. Balconi (SMS, Milan, Italy); A. Mattiussi, A. Antonini, (Azienda Ospedaliera Instituti Clinica di Perfezionamento, Parkinson Centre, Milan, Italy); C. Castiglioni, E. Battaglia (Medicasa, Milan, Italy); F. Moggio, M. Mercinelli (Telcom Italia Lab, Turin, Italy); W. Droogne, F. Van de Werf (Katholieke Universiteit Leuven, Leuven, Belgium); P. Sylvester, N. Pougetoux (Edelweb, Paris, France).

\section{REFERENCES}

1 Murray CJ, Lopez AD. Mortality by cause for eight regions of the world. Global Burden of Disease Study. Lancet 1997; 349: 1269-1276.

2 Pauwels RA, Buist AS, Calverley PM, Jenkins CR, , Hurd SS; GOLD Scientific Committee. Global strategy for the diagnosis, management, and prevention of chronic obstructive pulmonary disease. NHLBI/WHO Global Initiative for Chronic Obstructive Lung Disease (GOLD) Workshop summary. Am J Respir Crit Care Med 2001; 163: 1256-1276.

3 Rodriguez-Roisin R. Toward a consensus definition for COPD exacerbations. Chest 2000; 117: Suppl. 2, 398S-401S.

4 Domingo-Salvany A, Lamarca R, Ferrer M, et al. Healthrelated quality of life and mortality in male patients with chronic obstructive pulmonary disease. Am J Respir Crit Care Med 2002; 166: 680-685.

5 Seemungal TA, Donaldson GC, Paul EA, Bestall JC, Jeffries DJ, Wedzicha JA. Effect of exacerbation on quality of life in patients with chronic obstructive pulmonary disease. Am J Respir Crit Care Med 1998; 157: 1418-1422.

6 Donaldson GC, Seemungal TA, Bhowmik A, Wedzicha JA. Relationship between exacerbation frequency and lung function decline in chronic obstructive pulmonary disease. Thorax 2002; 57: 847-852. 
7 Garcia-Aymerich J, Monso E, Marrades RM, et al. Risk factors for hospitalization for a chronic obstructive pulmonary disease exacerbation. EFRAM study. Am J Respir Crit Care Med 2001; 164: 1002-1007.

8 Rich MW, Beckham V, Wittenberg C, Leven CL, Freedland KE, Carney RM. A multidisciplinary intervention to prevent the readmission of elderly patients with congestive heart failure. N Engl J Med 1995; 333: 1190-1195.

9 Stewart S, Vandenbroek AJ, Pearson S, Horowitz JD. Prolonged beneficial effects of a home-based intervention on unplanned readmissions and mortality among patients with congestive heart failure. Arch Intern Med 1999; 159: 257-261.

10 Bourbeau J, Julien M, Maltais F, et al. Reduction of hospital utilization in patients with chronic obstructive pulmonary disease: a disease-specific self-management intervention. Arch Intern Med 2003; 163: 585-591.

11 Gallefoss F. The effects of patient education in COPD in a 1-year follow-up randomised, controlled trial. Patient Educ Couns 2004; 52: 259-266.

12 Hermiz O, Comino E, Marks G, Daffurn K, Wilson S, Harris M. Randomised controlled trial of home based care of patients with chronic obstructive pulmonary disease. BMJ 2002; 325: 938.

13 Monninkhof E, van der Valk $\mathrm{P}$, van der Palen J, van Herwaarden C, Zielhuis G. Effects of a comprehensive self-management programme in patients with chronic obstructive pulmonary disease. Eur Respir J 2003; 22: 815-820.
14 Dahlen I, Janson C. Anxiety and depression are related to the outcome of emergency treatment in patients with obstructive pulmonary disease. Chest 2002; 122: 1633-1637.

15 Hernandez C, Casas A, Escarrabill J, et al. Home hospitalisation of exacerbated chronic obstructive pulmonary disease patients. Eur Respir J 2003; 21: 58-67.

16 Wagner EH. Meeting the needs of chronically ill people. BMJ 2001; 323: 945-946.

17 Celli BR, MacNee W. Standards for the diagnosis and treatment of patients with COPD: a summary of the ATS/ ERS position paper. Eur Respir J 2004; 23: 932-946.

18 Roca J., Hernández C., Alonso A., et al. Home management of exacerbations of chronic obstructive pulmonary disease. In: Siafakas NM, Anthonisen NR, Georgopoulos D, eds. Acute Exacerbations of Chronic Obstructive Pulmonary Disease. New York, Marcel Dekker, 2004; pp. 531-550.

19 Garcia-Aymerich J, Barreiro E, Farrero E, Marrades RM, Morera J, Anto JM. Patients hospitalized for COPD have a high prevalence of modifiable risk factors for exacerbation (EFRAM study). Eur Respir J 2000; 16: 1037-1042.

20 Wilkinson TM, Donaldson GC, Hurst JR, Seemungal TA, Wedzicha JA. Early therapy improves outcomes of exacerbations of chronic obstructive pulmonary disease. Am J Respir Crit Care Med 2004; 169: 1298-1303.

21 Wagner $\mathrm{EH}$. The role of patient care teams in chronic disease management. BMJ 2000; 320: 569-572.

22 World Health Organization. Innovative Care for Chronic Conditions: Building Blocks for Action. WHO/NMC/CCH/ 02.01. 2002 WHO Library Cataloguing-in-Publication Data pp. $1-112$. 